\title{
“Step-clipping" method: a technique for detecting previously bleeding diverticula
}

Bleeding of colonic diverticula is a common complaint, but it remains challenging to treat endoscopically because of the difficulty in determining the responsible diverticulum, particularly if the bleeding stops spontaneously. Additionally, endoscopic examinations lasting >60 minutes are, in some cases, also problematic [1]. Thus, an innovative diagnostic technique is eagerly anticipated.

Extravasation, observed during contrast-enhanced computed tomography (CECT), is an important factor in the identification of a bleeding diverticulum. However, even when CECT shows extravasation, colonoscopy-based detection of the responsible diverticulum occurs in only $60 \%-68 \%$ of cases [2-4]. This is because colonoscopy does not provide the precise positioning provided by $\mathrm{CT}$, particularly if active bleeding is absent. Here, we report a case of diverticular bleeding in which the "step-clipping" method was used to identify the responsible diverticulum ( $\triangleright$ Video 1 ).

A 70-year-old woman was admitted with massive hematochezia. Upon admission, CECT was conducted and revealed numerous diverticula in the ascending colon and active bleeding from one of them ( $\triangleright$ Fig.1). Subsequently, colonoscopy identified a fresh pool of blood remaining in the ascending colon. However, the active bleeding had spontaneously stopped, making detection of the responsible diverticulum difficult. After minimal searching, we placed five marking clips, 2 inches apart, in the ascending colon (step-clipping method), and performed unenhanced CT ( $\mathrm{D}$ Fig. 2). The positional relationship between each clip and the responsible diverticulum, identified in the initial CECT study, was evaluated. The responsible diverticulum was found opposite the second clip, near the ileocecal bulb ( $\mathbf{F i g . 3}$ ). Based on this evaluation, colonoscopy successfully identified the responsible diverticulum within 1 minute.

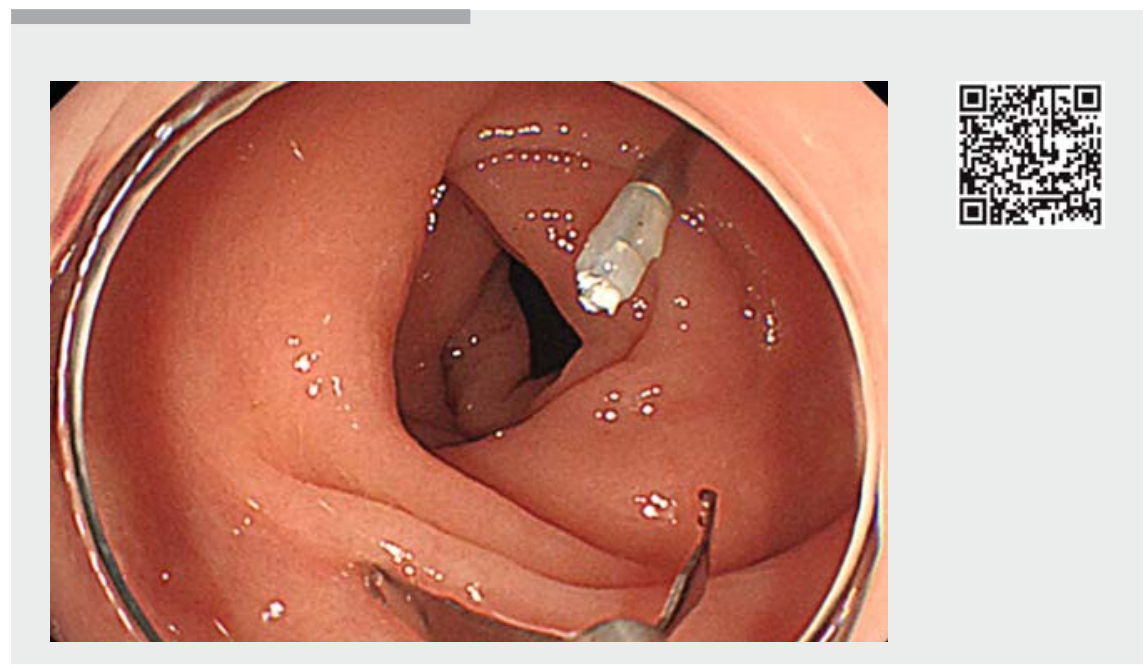

$\checkmark$ Video 1 Once bleeding has stopped, searching for the responsible diverticulum becomes difficult and time consuming. The step-clipping method provides precise positional information, allowing rapid, permanent hemostasis using endoscopic band ligation.

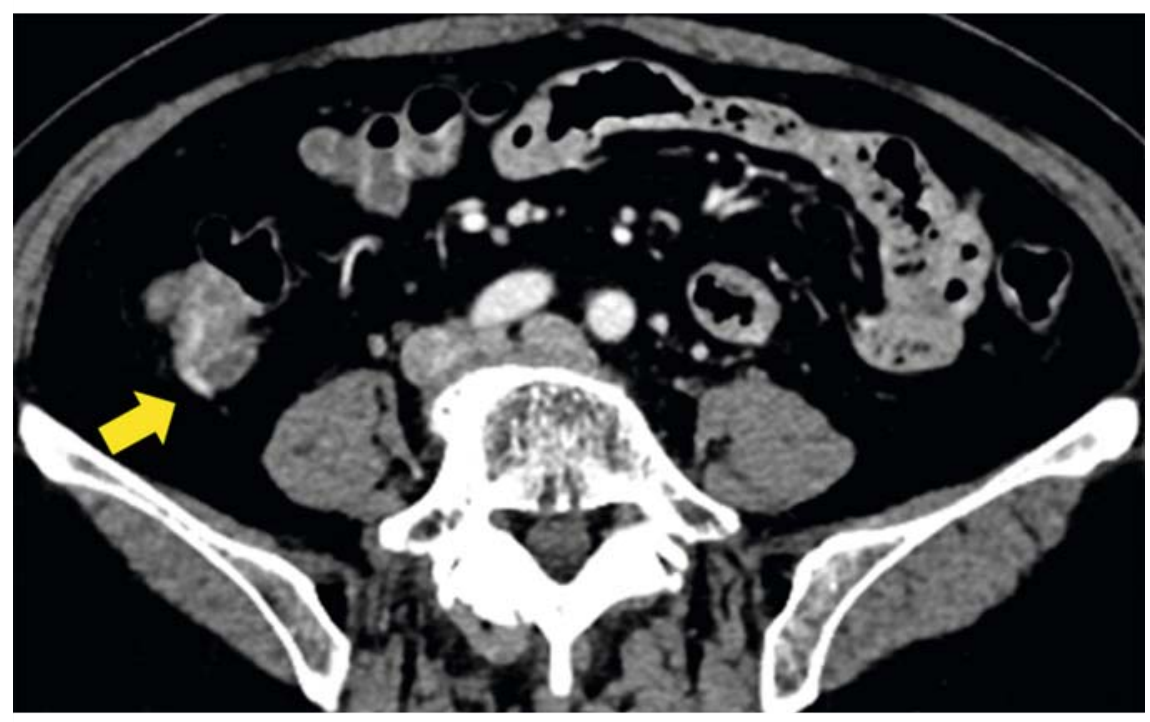

- Fig. 1 Extravasation from a dorsal diverticulum (arrow) on the ascending colon is shown in a contrast-enhanced computed tomography image, acquired on the day of admission.

Endoscopic band ligation [1] was performed to provide endoscopic hemostasis. An exposed vessel on the ligated diverticulum provided evidence of the cause of this bleeding incident. After ligation, there was no recurrence of bleed- ing, demonstrating the clinical feasibility of the step-clipping method.

Endoscopy_UCTN_Code_TTT_1AQ_2AZ 




Fig. 2 "Step-clipping" scheme. a Five clips are placed in the ascending colon, 2 inches apart. $\mathbf{b}$ The position of the five clips is schematically viewed via colonography.
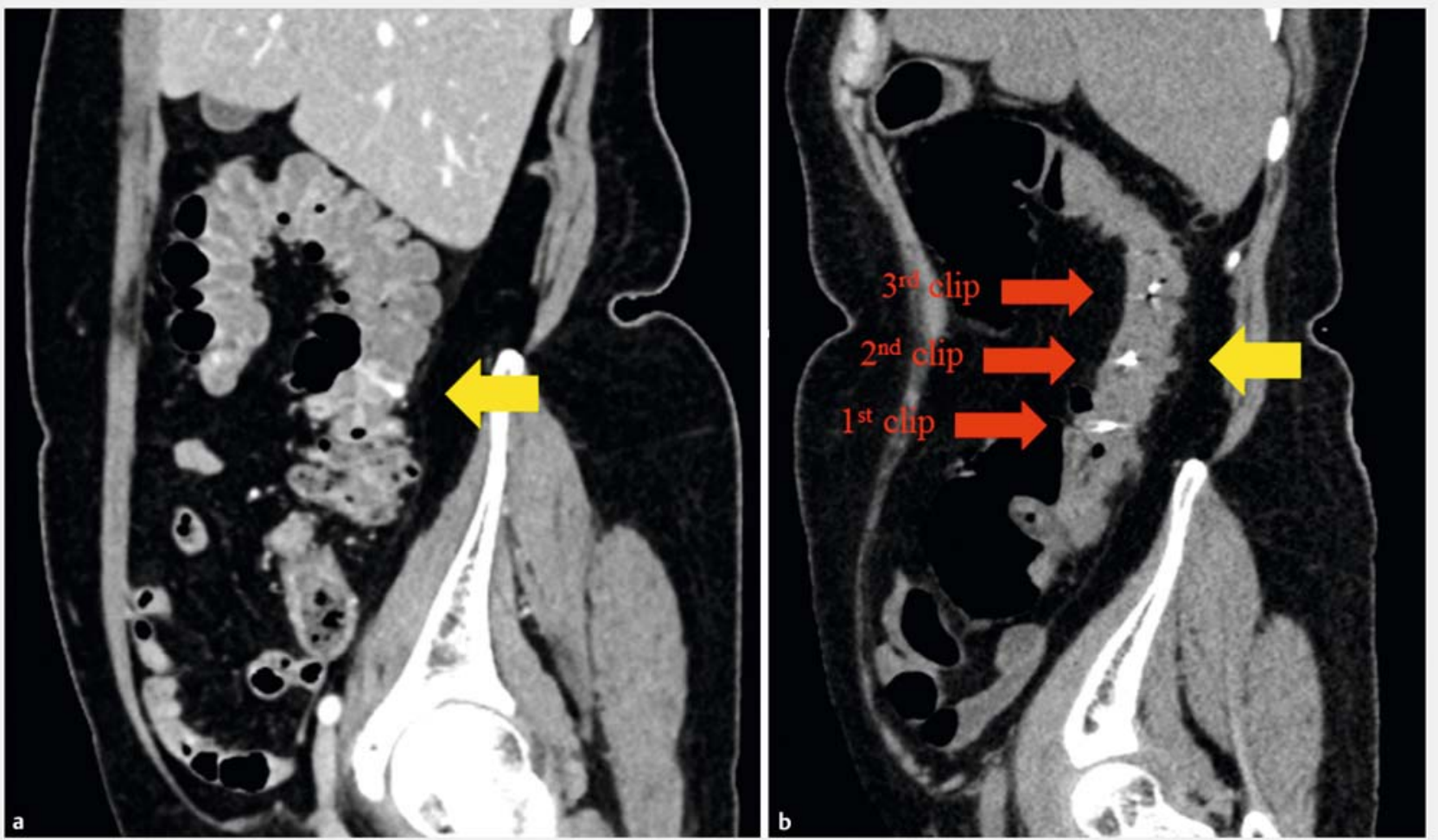

- Fig. 3 Comparison of sagittal computed tomography (CT) images before and after step-clipping. a Extavasation from the dorsal diverticulum in the ascending colon is evident (yellow arrow). b The responsible diverticulum is observed opposite the second clip (red arrow), near the ileocecal bulb, using unenhanced $\mathrm{CT}$. 
None

The authors

Hirosato Tamari ${ }^{1}$, Taiki Aoyama ${ }^{1}$, Shinji Nagata ${ }^{1}$, Kenjiro Shigita ${ }^{2}$, Naoki Asayama ${ }^{1}$, Akira Fukumoto ${ }^{2}$, Shinichi Mukai ${ }^{1}$

1 Department of Gastroenterology, Hiroshima City Asa Citizens Hospital, Hiroshima, Japan

2 Department of Endoscopy, Hiroshima City Asa Citizens Hospital, Hiroshima, Japan

\section{Corresponding author}

Taiki Aoyama, MD, PhD

Department of Gastroenterology, Hiroshima City Asa Citizens Hospital, 2-1-1 Kabeminami, Asakita-ku, Hiroshima 731-0293, Japan

Fax: +81-82-8141791

t-aoyama@asa-hosp.city.hiroshima.jp

\section{References}

[1] Shimamura Y, Ishii N, Omata F et al. Endoscopic band ligation for colonic diverticular bleeding: possibility of standardization. Endosc Int Open 2016; 4: E233 - E237

[2] Sugiyama T, Hirata Y, Kojima Y et al. Efficacy of contrast-enhanced computed tomography for the treatment strategy of colonic diverticular bleeding. Intern Med 2015; 54 : 2961-2967

[3] Nakatsu S, Yasuda H, Maeharta T et al. Urgent computed tomography for determining the optimal timing of colonoscopy in patients with acute lower gastrointestinal bleeding. Intern Med 2015; 54: 553 - 558

[4] Obana T, Fujita N, Sugita R et al. Prospective evaluation of contrast-enhanced computed tomography for the detection of colonic diverticular bleeding. Dig Dis Sci 2013; 58: $1985-1990$

\section{Bibliography}

DOI https://doi.org/10.1055/s-0043-120521

Published online: 25.10.2017

Endoscopy 2018; 50: E10-E12

(c) Georg Thieme Verlag KG

Stuttgart · New York

ISSN 0013-726X

\section{ENDOSCOPY E-VIDEOS}

https://eref.thieme.de/e-videos

回 Endoscopy E-Videos is a free 称 回望: on interesting cases and new techniques in gastroenterological endoscopy. All papers include a high quality video and all contributions are freely accessible online.

This section has its own submission website at

https://mc.manuscriptcentral.com/e-videos 